\title{
Clinical Outcomes of Transforaminal Endoscopic Lumbar Discectomy with or without Platelet-rich Plasma Injection in the Treatment of Lumbar Disc Herniation: A Prospective, Randomized, Controlled Pilot Study
}

\section{Yi Jiang}

Beijing Haidian Hospital https://orcid.org/0000-0002-6802-5189

\section{Rujun Zuo}

Beijing Haidian Section of Peking University Third Hospital: Beijing Haidian Hospital

\section{Shuai Yuan}

Beijing Haidian Section of Peking University Third Hospital: Beijing Haidian Hospital Jian Li

Beijing Haidian Section of Peking University Third Hospital: Beijing Haidian Hospital Chang Liu

Beijing Haidian Section of Peking University Third Hospital: Beijing Haidian Hospital Jiexun Zhang

Beijing Haidian Section of Peking University Third Hospital: Beijing Haidian Hospital

Ming Ma

Beijing Haidian Section of Peking University Third Hospital: Beijing Haidian Hospital

Dasheng Li

Beijing Haidian Section of Peking University Third Hospital: Beijing Haidian Hospital

Yong Hai ( $\nabla$ yong.hai@ccmu.edu.cn )

Beijing Chaoyang Hospital, Capital Medical University https://orcid.org/0000-0002-7206-325X

\section{Research article}

Keywords: Platelet-rich plasma, Transforaminal endoscopic lumbar discectomy, Annulus fibrosus remodeling, Magnetic resonance imaging, Randomized controlled trial

Posted Date: October 8th, 2020

DOI: https://doi.org/10.21203/rs.3.rs-86541/v1 
License: (c) (i) This work is licensed under a Creative Commons Attribution 4.0 International License. Read Full License 


\section{Abstract}

Background: This study aimed to evaluate the effectiveness of transforaminal endoscopic lumbar discectomy with or without platelet-rich plasma (PRP) in lumbar disc herniation treatment. Additionally, we aimed to identify whether the PRP injection could improve annulus fibrosus defects based on magnetic resonance imaging data.

Methods: Sixty patients with lumbar disc herniation were recruited and randomized to receive either transforaminal endoscopic lumbar discectomy with (PRP group, $n=30$ ) or without (control group, $n=30$ ) PRP injection. The visual analog scale score of back/leg pain and Oswestry disability index were recorded at 3 days, 6 months, and 1 year after surgery. Postoperative magnetic resonance imaging data were examined to determine the effects of PRP injection.

Results: No adverse events were reported in our study following the injection of PRP. Clinical improvement was noted in both groups. The visual analog scale score for leg pain at 3 days post-operation and back pain at 6 months post-operation were lower in the PRP group than those in the control group $(P<0.05)$. The magnetic resonance imaging data indicated the disc height decreased by $12 \%$ in the PRP group and $17 \%$ in the control group, whereas the spinal cross-sectional area increased by $15 \%$ in the PRP group and $6 \%$ in the control group after a 1-year follow-up. However, no statistically significant differences were found.

Conclusions: Transforaminal endoscopic lumbar discectomy with PRP injection around the annulus fibrosus defect was safe and could improve the clinical outcomes at the early postoperative stage. Moreover, PRP injection exerted no negative effects.

Trial Registration: ChiCTR 1800017228

\section{Introduction}

Worldwide, transforaminal endoscopic lumbar discectomy (TELD) is performed in patients with low back pain and/or sciatica due to lumbar disc herniation. TELD is clinically effective and results in a smaller incision size, less blood loss, reduced damage to the skeletal structure, and shorter hospitalization than open operation ${ }^{1}$. However, a recent meta-analysis reported that patients undergoing TELD required extended bed rest. Despite the symptoms being markedly improved, the repair of the annular defect after surgery by scar tissue required a longer period of time ${ }^{2}$. This finding seems to contradict the advantages of minimally invasive surgery. Annulus fibrosus (AF) defect that occurs after the removal of a herniated nucleus pulposus can influence reherniation ${ }^{3}$. The reherniation rate after TELD was higher than that after microdiscectomy in some studies $4 ; 5$. Numerous studies have focused on repairing AF defects with mechanistic devices in conventional operations ${ }^{6}$; however, such devices could not be performed under a full endoscopic system because of size limitations. Moreover, current research has tended to focus on 
biological remediation rather than mechanical repair ${ }^{7 ; 8}$. Thus, an effective and safe novel intervention strategy is warranted.

As an autologous derivative of whole blood containing a supraphysiological concentration of platelets, platelet-rich plasma (PRP) could deliver biomolecules to the target injured tissue with the modulation of inflammatory processes, thereby promoting healing and repair ${ }^{9}$. PRP application in disc degenerative disease and AF repair in vitro or in animal experiments has yielded promising results $7 ; 10 ; 11$. Few studies have explored whether radiculopathy and low back pain due to herniated nucleus pulposus could be improved by percutaneous PRP injection without serious complications ${ }^{12 ; 13}$. Basso et al. suggested that high-quality randomized clinical trials studying the potential of PRP injection were needed ${ }^{14}$. Moreover, no clinical study on the combination of TELD and PRP injection has been conducted.

Hence, this study aimed to assess the efficacy and safety of PRP injection in patients undergoing TELD and identify whether the PRP injection could improve AF defect healing.

\section{Methods}

\section{Study design}

We hypothesized that PRP injection administered in combination with TELD has clinical benefits and could improve AF remodeling. This prospective, open-label, randomized, controlled pilot trial included patients who underwent TELD with PRP injection between July 2018 and January 2019. This study was approved by the Institutional Review Board of Beijing Haidian Hospital (2018002) and registered on the Chinese Clinical Trial Registry (ChiCTR 1800017228). All patients provided written informed consent.

Power analysis was performed to calculate a sample size that could achieve $>80 \%$ power based on the visual analog scale (VAS) score difference in the pre-experiment. With an effect size of 0.77 , we estimated that at least 28 patients would be needed in each group (PRP and control groups).

Sixty patients were enrolled and randomly assigned to receive either TELD with PRP injection or TELD only, with a ratio of 1:1. Inclusion criteria were as follows: symptoms of low back pain and leg pain associated with herniated nucleus pulposus based on magnetic resonance imaging (MRI) data, singlelevel involvement without calcification, no lumbar surgery history, failed conservative treatment after 8 weeks, and platelet count $>150 \times 10^{9} / \mathrm{L}$. Patients who were pregnant, were receiving medications affecting platelet function, had coagulation disorders, had cauda equina syndrome, or were aged < 18 years or $>80$ years were excluded.

\section{PRP preparation}

PRP injection was performed using a sterile WEGO PRP preparation kit (Wego New Life Medical Devices Co., Ltd., Shandong, China). First, an anticoagulant was extracted using a $50 \mathrm{~mL}$ syringe, which 
sufficiently lubricated the inner wall of the syringe. Whole blood was collected from the median cubital vein. Subsequently, the blood was centrifuged at $2500 \mathrm{rpm}$ for $10 \mathrm{~min}$ to separate the whole blood and form a buffy coat layer containing platelets and white blood cells. Then, a $20 \mathrm{~mL}$ syringe was used to remove the excess red blood cells at $1 \mathrm{~mm}$ below the cone. Finally, a second centrifugation was conducted at $2750 \mathrm{rpm}$ for $10 \mathrm{~min}$ to further clear the buffy coat layer. The supernatant was removed with a $20 \mathrm{~mL}$ syringe. The pellet contained the PRP (Fig. 1) and was freshly prepared before use. The PRP volume for injection was $4 \mathrm{~mL}$.

\section{Surgical procedure}

All procedures were performed under local anesthesia administered by the same experienced orthopedic surgeon using the technique introduced by Hoogland ${ }^{15}$. Patients were placed in the prone position. An $18 \mathrm{G}$ needle was introduced from a skin entry point to the superior articular process of the lower involved vertebrae of the herniated disc under fluoroscopy monitoring. After the needle reached the target, a guide wire was inserted. A series of dilators were introduced, and a reamer was subsequently inserted through the cannula for foraminoplasty. While the neuroforamen was large enough for the working channel, the endoscope was introduced to observe the relationship of the nerve root and herniated nucleus pulposus under continuous irrigation. After the removal of the extruded or sequestrated fragment, the bulging annulus underneath the nerve root was also removed in cases where it was necessary. After draining out the fluids, the fresh PRP was mixed with $0.4 \mathrm{~mL}$ thrombin solution (1:10) to activate the platelets which simultaneously yielded a gel. This PRP gel mix was injected into the local site around the annuloplasty of patients in the PRP group (Fig. 2). In the control group, only discectomy was performed. The incision was closed without drainage in both groups.

\section{MRI technique}

Imaging of lumbar spines was obtained by a 1.5-Tesla MRI machine (HD-xt, GE Healthcare, USA). The postoperative MRI parameters were turbo echo T2-weighted (T2W) with 3000 ms repetition time and 75$85 \mathrm{~ms}$ echo time. Axial imaging was set with a slice thickness of $4 \mathrm{~mm}$ and an interslice gap of $0.4 \mathrm{~mm}$, $180 \times 180 \mathrm{~mm}$ field of view, and $400 \times 288$ matrix. Sagittal imaging was performed with a slice thickness of $3 \mathrm{~mm}$ without a gap, $280 \times 280 \mathrm{~mm}$ field of view, and $400 \times 312$ matrix.

\section{Measurements}

For the clinical evaluation, each patient completed a questionnaire consisting of standardized outcome assessments at baseline and at 3 days, 6 months, and 1 year after surgery; all patients returned for a clinical follow-up. Data including a VAS score of back pain and leg pain and Oswestry disability index (ODI) were obtained. 
Postoperative MRI parameters of the patients at 3 days and 1 year after surgery were assessed by one spine surgeon and one radiologist and compared between the two groups. Disc height (DH) was computed as the mean of the anterior and posterior DH from MRI data. Spine cross-sectional area (SCSA) was calculated on the axial cut of the T2W image using the miPlatform 3.0 software (Hinacom Software and Technology, Beijing, China). The SCSA was measured in the lower endplate plane, midline plane, and upper endplate plane, and the average was obtained.

\section{Statistical analysis}

Measurement data were expressed as mean \pm standard deviation. Categorical variables were presented as frequencies and percentages. Normally distributed data such as age, platelet count, DH, and SCSA were analyzed by the independent sample t-test, and the Mann-Whitney $\mathrm{U}$ test was used to analyze the difference in VAS and ODI. The significance of the differences in sex, surgical levels, and annular defect type was analyzed using the chi-square test. Interobserver reliability was calculated using the intraclass correlation coefficient (ICC). Analyses were performed using SPSS version 19.0 (IBM Corp. Armonk, NY). A $P<0.05$ was considered statistically significant.

\section{Results}

All patients completed the 1 year of follow-up. The mean hospital stay after surgery was $3.4 \pm 1.8$ days (range 3-5 days). Clinical data were analyzed in all 60 patients, whereas MRI data were only extracted from 20 patients in each group because some patients did not complete the MRI examination. No significant differences in baseline characteristics between the two groups were noted (Table 1). 
Table 1

Patient demographics, presentation, and procedural information (mean \pm SD)

\begin{tabular}{|c|c|c|c|}
\hline Characteristics & $\begin{array}{l}\text { PRP group } \\
n=30\end{array}$ & $\begin{array}{l}\text { Control group } \\
n=30\end{array}$ & $P$ value \\
\hline Age & $44.9 \pm 15.2$ & $52.5 \pm 14.7$ & 0.053 \\
\hline \multicolumn{4}{|l|}{ Sex } \\
\hline Male & $17(56.7 \%)$ & $18(60 \%)$ & 0.793 \\
\hline Female & $13(43.3 \%)$ & $12(40 \%)$ & \\
\hline Levels & & & 0.654 \\
\hline L3/L4 & $3(10 \%)$ & $4(13.3 \%)$ & \\
\hline L4/L5 & $18(60 \%)$ & $20(66.7 \%)$ & \\
\hline L5/S1 & $9(30 \%)$ & $6(20 \%)$ & \\
\hline Platelet levels( $\left.\times 10^{9} / \mathrm{L}\right)$ & $217.0 \pm 52.1$ & $235.9 \pm 65.9$ & 0.225 \\
\hline Annulus & & & 0.406 \\
\hline Natural tear only & $6(20 \%)$ & $7(23 \%)$ & \\
\hline Annulotomy only & $15(50 \%)$ & $10(33 \%)$ & \\
\hline Natural tear with annulotomy & $9(30 \%)$ & $13(43 \%)$ & \\
\hline \multicolumn{4}{|l|}{ Preoperative VAS Score } \\
\hline Leg pain & $6.83 \pm 1.44$ & $6.93 \pm 1.96$ & 0.124 \\
\hline Back pain & $2.43 \pm 1.17$ & $2.83 \pm 2.32$ & 0.822 \\
\hline Preoperative ODI (\%) & $52.83 \pm 20.09$ & $50.80 \pm 26.97$ & 0.482 \\
\hline
\end{tabular}

Improvement in the VAS score for leg pain at 3 days postoperatively was better in the PRP group than that in the control group $(P=0.012)$. However, no significant difference between the groups was observed at 6 months or 1 year postoperatively (all $P>0.05$ ). The VAS score for back pain in the PRP group significantly decreased 6 months after surgery $(P=0.021)$ compared to the control group. However, no difference between the groups was noted at 1 year after surgery. Moreover, no statistically significant difference in ODI scores between the two groups was observed at each time point $(P>0.05)$ (Table 2). 
Table 2

Comparison of clinical outcomes at 3 days, 6 months, and 1 year post-operation $($ mean $\pm \mathrm{SD})$

\begin{tabular}{|llll|}
\hline Characteristics & $\begin{array}{l}\text { PRP group } \\
\mathbf{n = 3 0}\end{array}$ & $\begin{array}{l}\text { Control group } \\
\mathbf{n}=\mathbf{3 0}\end{array}$ & Pvalue \\
\hline VAS Score & & & \\
\hline Leg pain at 3 days & $1.07 \pm 0.87$ & $1.67 \pm 0.84$ & $0.012^{*}$ \\
\hline Back pain at 3 days & $2.03 \pm 0.56$ & $2.20 \pm 0.76$ & 0.442 \\
\hline Leg pain at 6 months & $1.20 \pm 1.38$ & $1.27 \pm 1.02$ & 0.522 \\
\hline Back pain at 6 months & $1.23 \pm 0.63$ & $1.63 \pm 0.72$ & $0.021^{*}$ \\
\hline Leg pain at 1 year & $0.80 \pm 1.03$ & $1.03 \pm 0.93$ & 0.253 \\
\hline Back pain at 1 year & $1.47 \pm 0.78$ & $1.27 \pm 0.79$ & 0.263 \\
\hline ODI (\%) & & & 0.391 \\
\hline 6 months & $8.80 \pm 10.06$ & $11.30 \pm 11.36$ & 0.828 \\
\hline 1 year & $9.30 \pm 14.33$ & $7.60 \pm 8.41$ & \\
\hline SD: standard deviation, VAS: visual analogue scale, ODI: Oswestry disability index & & \\
\hline *P < 0.05 & & & \\
\hline
\end{tabular}

Mean $\mathrm{DH}$ at 3 days and 1 year after surgery was $8.6 \pm 1.7 \mathrm{~mm}$ and $7.4 \pm 1.5 \mathrm{~mm}$ in the PRP group, respectively, and $8.8 \pm 1.4 \mathrm{~mm}$ and $7.4 \pm 1.5 \mathrm{~mm}$ in the control group $(P>0.05)$. The difference value of $\mathrm{DH}$ and the decrease rate were $1.1 \pm 1.1 \mathrm{~mm}$ and $12 \%$, respectively, in the PRP group and $1.4 \pm 1.3 \mathrm{~mm}$ and $17 \%$, respectively, in the control group $(P>0.05)$. In addition, the mean SCSA at 3 days and 1 year postoperatively was $178.5 \pm 58.6 \mathrm{~mm}^{2}$ and $205.0 \pm 68.3 \mathrm{~mm}^{2}$, respectively, in the PRP group and $164.0 \pm$ $29.2 \mathrm{~mm}^{2}$ and $173.0 \pm 38.6 \mathrm{~mm}^{2}$, respectively, in the control group $(P>0.05)$. The difference value of SCSA and the increase rate were $26.5 \pm 21.9 \mathrm{~mm}^{2}$ and $15 \%$, respectively, in the PRP group and $9.0 \pm 30.7$ $\mathrm{mm}^{2}$ and $6 \%$ in the control group $(P>0.05)$ (Table 3$)$. No statistically significant differences in the data were found. 
Table 3

Comparison of MRI features at 3 days and 1 year post-operation

\begin{tabular}{|llll|}
\hline Characteristics & $\begin{array}{l}\text { PRP group } \\
\mathbf{n}=\mathbf{2 0}\end{array}$ & $\begin{array}{l}\text { Control group } \\
\mathbf{n}=\mathbf{2 0}\end{array}$ & Pvalue \\
\hline DH (mm) & & & \\
\hline 3 days & $8.6 \pm 1.7$ & $8.8 \pm 1.4$ & 0.455 \\
\hline 1 year & $7.4 \pm 1.5$ & $7.4 \pm 1.7$ & 0.774 \\
\hline Difference value & $1.1 \pm 1.1$ & $1.4 \pm 1.3$ & 0.872 \\
\hline Change rate (\%) & $12.3 \pm 12.1$ & $16.5 \pm 13.6$ & 0.943 \\
\hline SCSA (mm ${ }^{2}$ ) & & & \\
\hline 3 days & $178.5 \pm 58.6$ & $164.0 \pm 29.2$ & 0.050 \\
\hline 1 year & $205.0 \pm 68.3$ & $173.0 \pm 38.6$ & 0.089 \\
\hline Difference value & $26.5 \pm 21.9$ & $9.0 \pm 30.7$ & 0.29 \\
\hline Change rate (\%) & $14.8 \pm 12.3$ & $6.3 \pm 18.3$ & 0.102 \\
\hline SD: standard deviation, DH: disc height, SCSA: spinal cross-sectional area \\
\hline
\end{tabular}

Interrater reliability between two observers was calculated with ICCs. The ICCs of DH and SCSA were 0.826 to 0.975 , which indicates excellent reliability.

None of the patients with PRP injection exhibited neurological deterioration, symptoms of discitis, infection, hematoma, or deep vein thrombosis, which is consistent with findings from previous studies ${ }^{12}$; 13; 16 . Nonetheless, recurrent herniation was found in one patient each in the PRP and control groups.

\section{Discussion}

A lumbar disc herniation leads to radiating pain, and decompression is critical for the treatment. TELD contributes to efficient pain relief because of proper decompression and sustained irrigation which can decrease local inflammation of the affected site. A herniated nucleus compresses the nerve root resulting in inflammation, and inflammatory factors are one of the primary causes of radiating pain in this condition. The diverse factors released by PRP promote cell survival and proliferation, downregulation of pro-inflammatory cytokines, suppression of tumor necrosis factor KB pathways and endogenous cannabinoid systems, subchondral bone homeostasis, and bone mineralization ${ }^{17}$. Using an animal experiment, Kamoda et al. reported that PRP can reduce inflammatory calcitonin gene-related peptide levels in sensory neurons innervating the discs ${ }^{18}$. In a recent study ${ }^{19}$, perineural PRP injection could promote improvement in peripheral neurofunction with a decreasing proportion of inflammatory neuropeptides. Bhatia and Chopra recommended using PRP injection for treating patients with prolapsed 
intervertebral disc via the interlaminar lumbar epidural approach, which could improve both pain and function ${ }^{16}$. In our study, we demonstrated that PRP injection could decrease pain by reducing inflammatory factors around the lesion. This was observed at an early stage and a significant improvement in the VAS score for leg pain was noted in the PRP group compared to the control group at 3 days postoperatively $(P=0.012)$. Despite the good clinical results, further research to better determine the effects of PRP on neural function adjustment is needed.

In a previous prospective clinical trial ${ }^{20}$, the success rate of intradiscal PRP injection for discogenic low back pain was $47 \%$ at 6 months after administration. Another study suggested that intradiscal PRP injection could improve symptomatic degenerative intervertebral discs, which was effective for 1 year ${ }^{21}$. Using the same method of PRP injection, Cheng et al. showed a statistically and clinically significant improvement in pain and function at 5-9 years post-injection ${ }^{22}$. In our series, the VAS score for back pain at 6 months after surgery significantly improved in the PRP group. Regarding function, no significant difference in ODI score between the two groups was noted at any time point. The PRP infiltration effects on extracellular matrix synthesis, anti-inflammatory mechanisms, analgesia, and subchondral bone homeostasis may contribute to restore disc and bone homeostasis. All of these could stimulate the endogenous repair machinery and reduce the pain.

Residual annulus observed via MRI is common in the early postoperative period after TELD and gradually decreases over time ${ }^{23}$. The $32 \%$ increase in postoperative SCSA in the 1-year follow-up indicates that the endogenous repair is connected to the residual annulus. In our study, the SCSA increased from $178.5 \pm$ $58.6 \mathrm{~mm}^{2}$ to $205.0 \pm 68.3 \mathrm{~mm}^{2}$ in the PRP group and from $164.0 \pm 29.2 \mathrm{~mm}^{2}$ to $173.0 \pm 38.6 \mathrm{~mm}^{2}$ in the control group (Fig. 3). Although the change rate was higher in the PRP group (15\%) than that in the control group (6\%), no statistically significant difference was found.

Generally, PRP injection could improve defects and promote wound healing directly at the site of injury. PRP has proliferative effects on AF cells and is able to increase extracellular matrix production in vitro. PRP supplementation creates a gel-like structure, which affects the morphology of AF cells, and, therefore, may be considered to promote AF repair ${ }^{7}$. However, AF defect progression after discectomy is a complex biological process. Therefore, advanced techniques ${ }^{24 ;} 25$ such as microcomputed tomography or fractional anisotropy in diffusion tensor imaging should be explored to confirm the effectiveness of PRP injection in annulus remodeling.

Gui et al. reported that PRP injection could prevent a decrease in DH in a rabbit model ${ }^{10}$. However, in our study, DH decreased in both groups at 1 year after TELD. DH decreased by $12 \%$ in the PRP group and $17 \%$ in the control group, although no significant difference between the groups was noted (Fig. 4). We suggest that differences in posture and gait between the animal model and humans account for the different results.

Although a significant reparative effect based on image parameters was not observed in this study, our findings suggested that PRP injection has no negative effect, and no adverse events were observed. 
Advanced clinical trial design is needed to determine further the benefit of PRP application.

\section{Limitations}

This study has some limitations. Compared to clinical information, MRI data were incomplete. The orientation of the axial cut of the $\mathrm{T} 2 \mathrm{~W}$ image at 1 year was not precisely in accordance with the previous one. Measurement bias was inevitable, although two independent researchers were involved in this study to improve the accuracy of the measurements. Lastly, the sample size in this study was small. Hence, further investigation with larger sample size and long-term observation is needed.

\section{Conclusion}

To our knowledge, this is the first clinical trial of TELD performed in conjunction with PRP injection for the treatment of patients with lumbar disc herniation. PRP injection following TELD is a safe procedure and could improve the clinical outcomes at the early postoperative stage.

\section{Abbreviations}

AF: annulus fibrosus; DH: disc height; ICC: intraclass correlation coefficient; MRI: magnetic resonance imaging; ODI: Oswestry disability index; PRP: platelet-rich plasma; SCSA: spine cross-sectional area; T2W: T2-weighted; TELD: transforaminal endoscopic lumbar discectomy; VAS: visual analog scale

\section{Declarations}

\section{Ethics approval and consent to participate}

This study was approved by the Institutional Review Board of Beijing Haidian Hospital (2018002) and registered on the Chinese Clinical Trial Registry (ChiCTR 1800017228). All patients provided written informed consent.

\section{Consent for publication}

Not applicable.

\section{Availability of data and materials}

All data generated or analyzed during this study are included in this published article.

\section{Competing interests}


None.

\section{Funding}

This study was supported by Capital's Funds for Health Improvement and Research (grant number 20183-7041).

\section{Authors' contributions}

Conception and design: Yi Jiang. Acquisition of data: Shuai Yuan, Jian Li, Dasheng Li. Analysis and interpretation of data: Jiexun Zhang, Ming Ma. Drafting the article: Rujun Zuo. Critically revising the article: Yong Hai.

\section{Acknowledgements}

We would like to thank Editage (www.editage.cn) for English language editing.

\section{References}

1. Ahn Y, Lee SG, Son S, Keum HJ. Transforaminal endoscopic lumbar discectomy versus open lumbar microdiscectomy: A comparative cohort study with a 5-year follow-up. Pain Physician. 2019;22:295304.

2. Ding W, Yin J, Yan T, Nong L, Xu N. Meta-analysis of percutaneous transforaminal endoscopic discectomy vs. fenestration discectomy in the treatment of lumbar disc herniation. Orthopade. 2018;47:574-84.

3. Miller LE, Allen RT, Duhon B, Radcliff KE. Expert review with meta-analysis of randomized and nonrandomized controlled studies of Barricaid annular closure in patients at high risk for lumbar disc reherniation. Expert Rev Med Devices. 2020;17:461-9.

4. Gibson JNA, Subramanian AS, Scott CEH. A randomised controlled trial of transforaminal endoscopic discectomy vs microdiscectomy. Eur Spine J. 2017;26:847-56.

5. Liu X, Yuan S, Tian Y, Wang L, Gong L, Zheng Y, et al. Comparison of percutaneous endoscopic transforaminal discectomy, microendoscopic discectomy, and microdiscectomy for symptomatic lumbar disc herniation: minimum 2-year follow-up results. J Neurosurg Spine. 2018;28:317-25.

6. Ammerman J, Watters WC, Inzana JA, Carragee G, Groff MW. Closing the treatment gap for lumbar disc herniation patients with large annular defects: A systematic review of techniques and outcomes in this high-risk population. Cureus. 2019;11:e4613.

7. Pirvu TN, Schroeder JE, Peroglio M, Verrier S, Kaplan L, Richards RG, et al. Platelet-rich plasma induces annulus fibrosus cell proliferation and matrix production. Eur Spine J. 2014; 23:745-53. 
8. Jin L, Shimmer AL, Li X. The challenge and advancement of annulus fibrosus tissue engineering. Eur Spine J. 2013;22:1090-100.

9. Wu PI, Diaz R, Borg-Stein J. Platelet-rich plasma. Phys Med Rehabil Clin N Am. 2016;27:825-53.

10. Gui K, Ren W, Yu Y, Li X, Dong J, Yin W. Inhibitory effects of platelet-rich plasma on intervertebral disc degeneration: a preclinical study in a rabbit model. Med Sci Monit. 2015;21:1368-75.

11. Gelalis ID, Christoforou G, Charchanti A, Gkiatas I, Pakos E, Papadopoulos D, et al. Autologous platelet-rich plasma (PRP) effect on intervertebral disc restoration: an experimental rabbit model. Eur J Orthop Surg Traumatol. 2019;29:545-51.

12. Bise $S$, Dallaudiere B, Pesquer L, Pedram M, Meyer P, Antoun MB, et al. Comparison of interlaminar CT-guided epidural platelet-rich plasma versus steroid injection in patients with lumbar radicular pain. Eur Radiol. 2020;30:3152-60.

13. Kirchner F, Anitua E. Intradiscal and intra-articular facet infiltrations with plasma rich in growth factors reduce pain in patients with chronic low back pain. J Craniovertebr Junction Spine. 2016;7:250-6.

14. Basso M, Cavagnaro L, Zanirato A, Divano S, Formica C, Formica M, et al. What is the clinical evidence on regenerative medicine in intervertebral disc degeneration? Musculoskelet Surg. 2017;101:93-104.

15. Hoogland T, Schubert M, Miklitz B, Ramirez A. Transforaminal posterolateral endoscopic discectomy with or without the combination of a low-dose chymopapain: a prospective randomized study in 280 consecutive cases. Spine (Phila Pa 1976). 2006;31:E890-7.

16. Bhatia R, Chopra G. Efficacy of platelet rich plasma via lumbar epidural route in chronic prolapsed intervertebral disc patients-A pilot study. J Clin Diagn Res. 2016;10:UC05-07.

17. Kirchner F, Pinar A, Milani I, Prado R, Padilla S, Anitua E. Vertebral intraosseous plasma rich in growth factor (PRGF-Endoret) infiltrations as a novel strategy for the treatment of degenerative lesions of endplate in lumbar pathology: description of technique and case presentation. J Orthop Surg Res. 2020;15:72.

18. Kamoda H, Yamashita M, Ishikawa T, Miyagi M, Arai G, Suzuki M, et al. Platelet-rich plasma combined with hydroxyapatite for lumbar interbody fusion promoted bone formation and decreased an inflammatory pain neuropeptide in rats. Spine (Phila Pa 1976). 2012;37:1727-33.

19. Anjayani S, Wirohadidjojo YW, Adam AM, Suwandi D, Seweng A, Amiruddin MD. Sensory improvement of leprosy peripheral neuropathy in patients treated with perineural injection of plateletrich plasma. Int J Dermatol. 2014;53:109-13.

20. Levi D, Horn S, Tyszko S, Levin J, Hecht-Leavitt C, Walko E. Intradiscal platelet-rich plasma injection for chronic discogenic low back pain: Preliminary results from a prospective trial. Pain Med. 2016;17:1010-22.

21. Tuakli-Wosornu YA, Terry A, Boachie-Adjei K, Harrison JR, Gribbin CK, LaSalle EE, et al. Lumbar intradiskal platelet-rich plasma (PRP) injections: A prospective, double-blind, randomized controlled study. PM R. 2016;8:1-10. 
22. Cheng J, Santiago KA, Nguyen JT, Solomon JL, Lutz GE. Treatment of symptomatic degenerative intervertebral discs with autologous platelet-rich plasma: follow-up at 5-9 years. Regen Med. 2019;14:831-40.

23. Mahatthanatrakul A, Kotheeranurak V, Lin GX, Hur JW, Chung HJ, Kim JS. Comparative analysis of the intervertebral disc signal and annulus changes between immediate and 1-year postoperative MRI after transforaminal endoscopic lumbar discectomy and annuloplasty. Neuroradiology. 2019;61:411-9.

24. Senck S, Trieb K, Kastner J, Hofstaetter SG, Lugmayr H, Windisch G. Visualization of intervertebral disc degeneration in a cadaveric human lumbar spine using microcomputed tomography. J Anat. 2020;236:243-51.

25. Perri M, D'Elia M, Castorani G, Balzano RF, Pennelli A, Al-Badayneh B, et al. Assessment of lumbar disc herniaton using fractional anisotropy in diffusion tensor imaging along with conventional T2weighted imaging. Neuroradiol J. 2020;33:24-31.

\section{Figures}

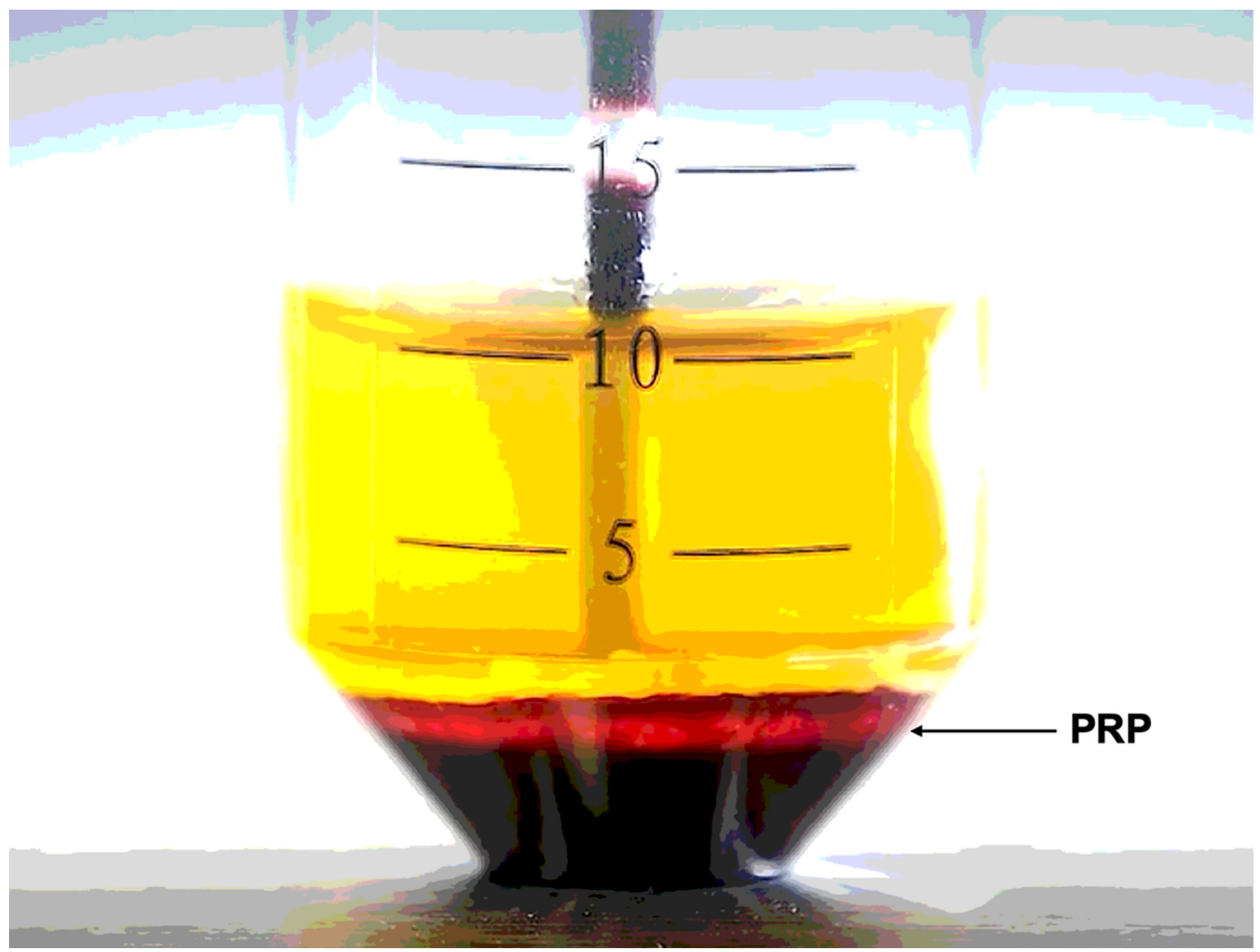




\section{Figure 1}

Platelet-rich plasma after the second centrifugation.

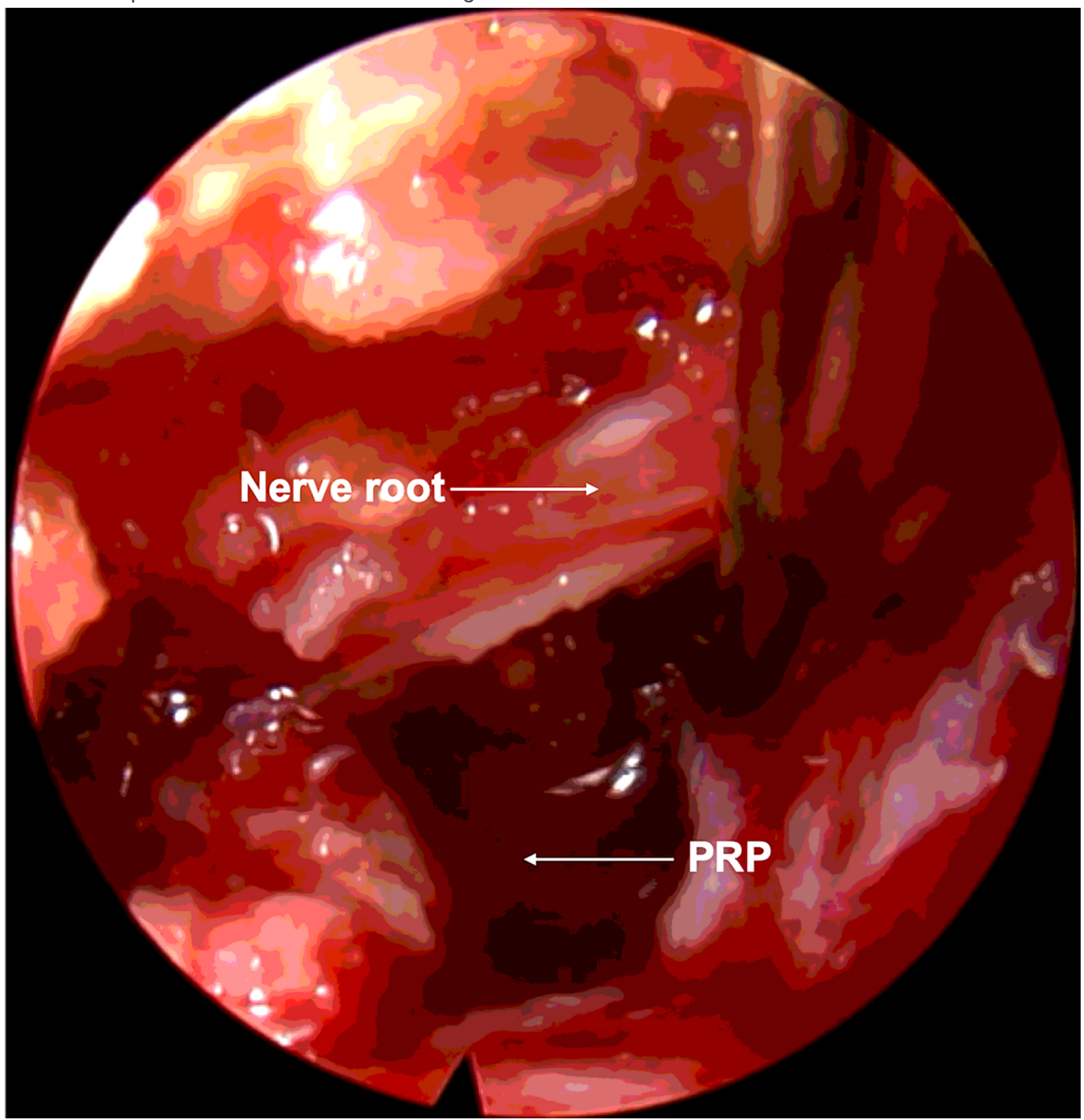

Figure 2

Platelet-rich plasma injection after transforaminal lumbar endoscopic discectomy. 


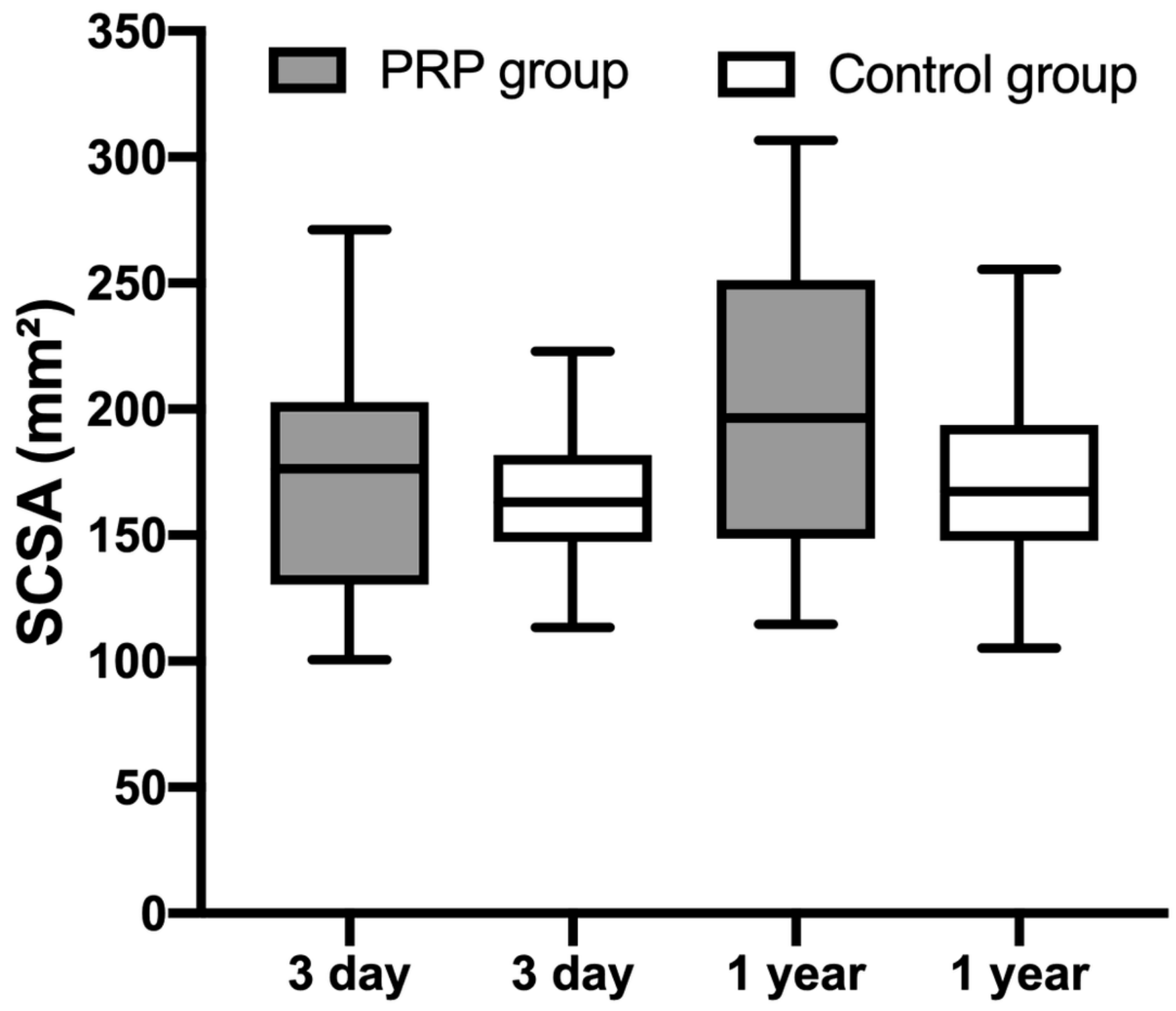

Figure 3

Comparison of spinal cross-sectional area (SCSA) on magnetic resonance images (MRI) at 3 days and 1 year post-operation. 


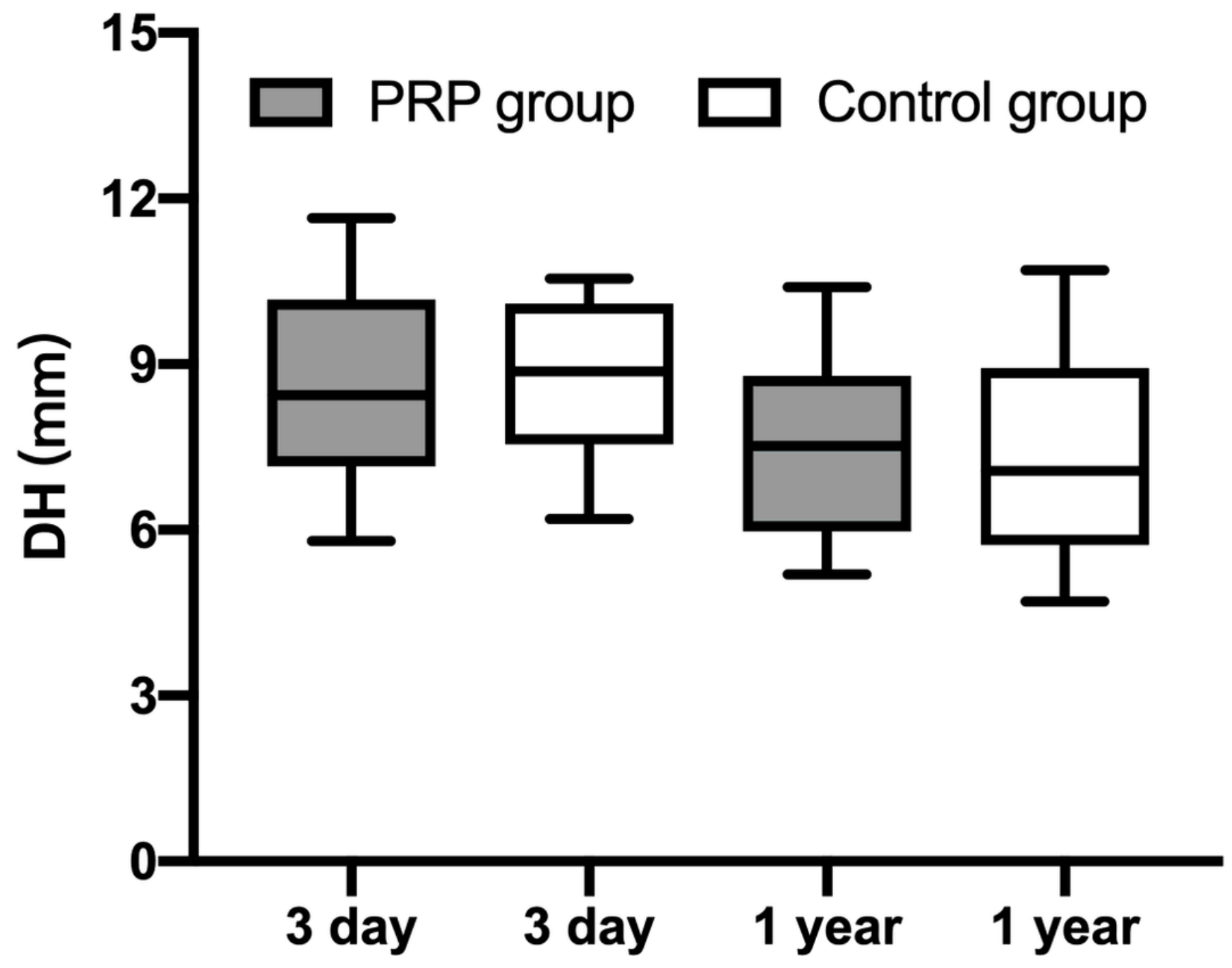

Figure 4

Comparison of disc height ( $\mathrm{DH})$ on magnetic resonance images (MRI) at 3 days and 1 year postoperation. 